\title{
Técnicas e parâmetros utilizados nos estudos de compatibilidade entre fungos entomopatogênicos e produtos fitossanitários ${ }^{1}$
}

\section{Techniques and parameters used in compatibility studies between entomopathogenic fungi and phytosanitary products ${ }^{1}$}

\author{
Roberta Zani da Silva ${ }^{2}$; Pedro Manuel de Oliveira Janeiro Neves 3 ; \\ Patricia Helena Santoro ${ }^{4}$
}

\begin{abstract}
Resumo
Os fungos são importantes agentes de controle biológico de pragas, pois podem infectar o inseto em diversos estágios de desenvolvimento. As interações entre estes microrganismos e os produtos fitossanitários devem ser consideradas nos programas de Manejo Integrado de Pragas (MIP), assim como já é realizado para insetos predadores e parasitóides. A adoção de produtos seletivos aos fungos entomopatogênicos possibilitará maior eficiência na conservação do entomopatógeno, incrementando o controle biológico. Os estudos de seletividade/compatibilidade são, na sua grande maioria, realizados em testes in vitro pela facilidade em relação aos estudos a campo. Entretanto, nos testes in vitro os métodos empregados no contato entre os produtos e os fungos entomopatogênicos e os parâmetros avaliados são bastante diferentes o que dificulta a comparação entre os resultados. Assim, esta revisão discute as principais técnicas e parâmetros utilizados nestes testes e sugere diretrizes para o estabelecimento de protocolos que facilitarão a comparação de resultados e disponibilizarão informações importantes para recomendação de produtos fitossanitários no desenvolvimento de estratégias de controle de pragas.
\end{abstract}

Palavras-chave: Entomopatógenos, agroquímicos, seletividade

\begin{abstract}
Fungi are important biological control agents of pests, because they can infect the insect at several developmental stages. The interactions between these microorganisms and phytosanitary products must be taken into account in Integrated Pest Management (IPM) programs, much the same way as it is done for predator and parasitoid insects. The adoption of products that are selective to entomopathogenic fungi will provide greater efficiency in the preservation of the entomopathogen, enhancing biological control. Selectivity/compatibility studies are, for the most part, conducted in the form of in vitro tests because of their convenience in relation to field studies. However, the methods used in in vitro tests for obtaining contact between products and entomopathogenic fungi, in addition to the evaluated parameters,
\end{abstract}

1 Parte integrante da Dissertação de Mestrado da primeira autora, apresentada ao Programa de Pós-Graduação em Agronomia da Universidade Estadual de Londrina.

2 Engenheira Agrônoma, Mestre, Doutoranda em Agronomia da Universidade Estadual de Londrina, Bolsista CNPq. e-mail: robertazani@dilk.com.br.

3 Engenheiro Agrônomo, Doutor, Professor do Departamento de Agronomia da Universidade Estadual de Londrina, Bolsista CNPq e-mail: pedroneves@uel.br.

4 Engenheira Agrônoma, Mestranda em Agronomia da Universidade Estadual de Londrina, Bolsista CAPES.email:patysantoro@zipmail.com.br.

* Autor para correspondência. 
are quite different, which make the comparison between results a difficult undertaking. In this review we therefore discuss the most important techniques and parameters used in these tests and suggest guidelines for protocol establishment. These protocols will facilitate the comparison of results and make important information available for the recommendation of phytosanitary products in the development of pest control strategies.

Key words: Entomopathogens, agrochemicals, selectivity

\section{Introdução}

A conservação dos fungos entomopatogênicos dentro do agroecossistema é considerada a estratégia mais simples e econômica de utilização destes microrganismos que pode ser realizada, entre outras técnicas, através da utilização de produtos fitossanitários seletivos.

As interações entre os fungos entomopatogênicos e os produtos fitossanitários podem ser positivas, quando ocorre uma ação sinérgica ou aditiva entre o patógeno e o produto, ou negativas, quando ocorre a inibição de um dos componentes, geralmente a do patógeno. Estes produtos, principalmente os fungicidas, inibem, na sua grande maioria, a germinação dos esporos dos fungos entomopatogênicos diminuindo o potencial de inóculo. Assim, as interações, principalmente as negativas, devem ser consideradas nos programas de Manejo Integrado de Pragas, pois, quanto mais seletivo (compatível) for o produto químico mais eficiente será a conservação do entomopatógeno. Este aspecto é mais importante em agroecossistemas onde o fungo entomopatogênico é um importante fator de redução populacional de insetos, sendo considerado um inimigo natural-chave.

Os estudos de compatibilidade entre produtos fitossanitários e fungos entomopatogênicos têm sido realizados desde os primeiros programas de controle com estes patógenos (HALL; DUNN, 1959; JAQUES; PATTERSON, 1962; IGNOFFO et al., 1975; ALVES; RIGITANO; CAMARGO, 1980), entretanto, as técnicas utilizadas são geralmente muito diversas, principalmente no método de contado entre o fungo e o produto, nas doses utilizadas e nos parâmetros avaliados, dificultando a comparação dos resultados obtidos nos diferentes estudos.
Poucas são as recomendações e tentativas de padronização dos testes de compatibilidade. A IOBC - International Organization for Biological Control (HOKKANEN; KOTILUOTO, 1992) sugere uma metodologia para testes de compatibilidade, em laboratório, semi-campo e campo. Nesta metodologia, em laboratório, estuda-se o crescimento vegetativo e a produção de esporos em meio batata-dextroseágar (BDA) contendo o produto. Com os esporos produzidos quantifica-se a viabilidade e a virulência em larvas de Tenebrio molitor. Entretanto, nesta técnica não é avaliada a inibição da germinação dos esporos do fungo.

Outra metodologia padrão é proposta por Alves, Moino e Almeida (1998), segundo a qual os produtos químicos são adicionados em concentrações préestabelecidas (geralmente as recomendadas em campo) ao meio de cultura fundido, ainda não solidificado. Os parâmetros avaliados são diâmetro de colônias (crescimento vegetativo) e a produção de esporos (esporulação). Os autores sugerem ainda a classificação dos produtos quanto a sua toxicidade ao patógeno, baseada na fórmula $\mathrm{T}=\{20(\mathrm{CV})+$ 80 (ESP) $\} / 100$, onde os valores para $\mathrm{CV}$ (crescimento vegetativo) e ESP (esporulação) são obtidos em relação à testemunha $(100 \%)$. $\mathrm{O}$ valor $\mathrm{T}$ define a toxicidade dos produtos nos intervalos: 0 a $30=$ muito tóxico; 31 a $45=$ tóxico; 46 a $60=$ moderadamente tóxico; $>60=$ compatível.

A metodologia proposta por Alves, Moino e Almeida (1998) é a mais utilizada in vitro e tem sido adotada em muitos experimentos, principalmente no Brasil (MOINO; ALVES, 1998; BATISTA FILHO; ALMEIDA; LAMAS, 2001), porém esta metodologia, assim como a da IOBC, não considera o efeito direto dos produtos fitossanitários sobre a 
germinação dos esporos de fungos entomopatogênicos, considerada por muitos autores como o principal fator na compatibilidade (LORIA; GALAINI; ROBERTS, 1983; ANDERSON; ROBERTS, 1983; MALO, 1993; HIROSE et al., 2001; NEVES et al., 2001; OLIVEIRA;NEVES; KAWAZOE, 2003).

\section{Desenvolvimento}

Técnicas de Contato Entre Produtos Fitossanitários e Fungos Entomopatogênicos

Incorporação do produto químico ao meio de cultura

Nesta técnica os produtos são adicionados aos meios de cultura sólidos, quando este ainda se encontra líquido na faixa de temperatura de 40 a $50^{\circ} \mathrm{C}$ ou diretamente no meio líquido. A maioria dos autores utilizou esta técnica para estudar a ação dos produtos químicos sobre os patógenos.

Cadatal e Gabriel (1970) realizaram estudos sobre a compatibilidade de inseticidas e fungicidas no crescimento vegetativo e esporulação de Beauveria bassiana, Metarhizium anisopliae e Entomophthora sp., assim como Olmert e Kenneth (1974) verificaram os efeitos de fungicidas, inseticidas e acaricidas sobre os fungos entomopatogênicos $B$. bassiana, Verticillium lecanii e Verticillium sp.

A incorporação do produto no meio foi também utilizada para estudar a compatibilidade de alguns herbicidas sobre o crescimento e esporulação in vitro dos fungos entomopatogênicos $B$. bassiana, Conidiobolus rhomboides, C. coronatus, $M$. anisopliae, P. farinosus e V. lecanii, por Poprawski e Majchrowicz (1995). Nesse estudo utilizou-se o ingrediente ativo dos produtos previamente dissolvidos em acetona, adicionandoos ao meio de cultura Sabouroud-Dextrose-Ágar (SDA). Ao contrário da maioria dos trabalhos, onde o fungo é inoculado diretamente sobre o meio, neste estudo foi retirado um pedaço de meio (1 $\mathrm{mm}$ de profundidade $\mathrm{x} 2 \mathrm{~mm}$ de diâmetro) do centro da placa formando-se um poço, onde se adicionou $0,1 \mathrm{~mL}$ de suspensão de fungo contendo aproximadamente 500 esporos viáveis.

A avaliação do crescimento micelial de $B$. bassiana em meio líquido Saboroud-Dextrose-Batata (SDB) contendo fungicidas e herbicidas, nas concentrações recomendadas para campo foi estudada por Todorova et al. (1998). O meio de cultura foi inoculado com uma suspensão do fungo ficando sob agitação por sete dias. Após este período comparou-se o peso da massa micelial dos tratamentos com a testemunha.

Observa-se que, mesmo nos estudos de incorporação dos produtos/formulações aos meios sólidos ou líquidos foram empregadas diferentes técnicas. Alguns autores utilizam o produto formulado, outros utilizam apenas o ingrediente ativo. Alguns inoculam os esporos sobre o meio de cultura, outros utilizam o método de poço.

É importante salientar que os testes realizados em laboratório devem simular o que pode ocorrer no campo. Sendo assim, estas técnicas de incorporação do produto químico ao meio de cultura podem prever o que aconteceria se o produto entrasse em contato com o fungo dentro do corpo do inseto. Entretanto, se este contato no interior do corpo do inseto ocorrer, esta técnica pode dar um resultado superestimado já que a quantidade de produto presente no inseto é provavelmente menor do que a utilizada nos testes. Também no caso de contato do inseto com o inseticidas, ocorrerá a morte do mesmo dificultando o desenvolvimento do patógeno devido à competição com bactérias saprofíticas que colonizarão mais rapidamente o corpo do inseto. No caso dos fungicidas e herbicidas, a morte do inseto não ocorrerá mas a concentração dos produtos na hemolinfa será provavelmente muito menor do que a utilizada nos testes in vitro. 
Discos impregnados com produto fitossanitário

Outra técnica utilizada nos estudos de compatibilidade é a de discos de papel de filtro impregnados com os produtos químicos e colocados sobre o meio de cultura sólido previamente inoculado com o entomopatógeno. Neste caso, a avaliação é feita indiretamente através do crescimento vegetativo medindo o halo de inibição formado (DOUGHERTY; REICHELDERFER; FAUST, 1971). Esta técnica parece ser mais adequada quando o entomopatógeno tem desenvolvimento saprofítico, o qual poderá ser inibido quando em contato com o produto presente no solo.

\section{Pulverização do produto}

Esta técnica apesar de ser a que melhor simula o que ocorre no campo é pouco citada em testes in vitro. Em estudo para comparar diferentes técnicas utilizadas na avaliação da compatibilidade de produtos químicos com B. bassiana Silva (2003) testou a pulverização dos produtos sobre o meio de cultura em placas de Petri, antes e após a inoculação do entomopatógeno.

Para avaliar o impacto de fungicidas sobre isolados de $B$. bassiana na indução de mortalidade de larvas Leptinotarsa decemlineata (Say), Jarussu, Groden e Zhang (1999) pulverizaram os produtos em folhas de batata em placas de Petri antes da pulverização do fungo $B$. bassiana e avaliaram a germinação dos esporos. Assim, puderam simular de forma mais adequada o que ocorre a campo, já que na maioria das vezes, os produtos fitossanitários são pulverizados/polvilhados sobre as plantas onde se encontram as estruturas (esporos) do patógeno.

\section{Mistura de esporos à calda de produto}

Nesta técnica os produtos e os patógenos são misturados, simulando uma aplicação conjunta dos mesmos. Esta técnica foi empregada para estudar a possível utilização conjunta de $B$. bassiana com produtos fitossanitários no manejo integrado de L. decemlineata (Say). Neste estudo foram comparadas 13 formulações de inseticidas, adicionando esporos do fungo à calda dos produtos. Após a mistura avaliou-se a inibição da germinação em diferentes intervalos de tempo (ANDERSON; ROBERTS, 1983). Esta técnica foi também utilizada por Hirose et al. (2001) para verificar o efeito de fertilizantes e óleo de nim (Azadirachta indica) sobre a germinação de esporos de $M$. anisopliae e B. bassiana.

Oliveira, Neves e Kawazoe (2003) utilizaram técnica semelhante no estudo da compatibilidade de diferentes produtos, utilizados na lavoura cafeeira, com B. bassiana.

A mistura dos esporos à calda do produto é uma simulação da mistura de formulações de entomopatógenos e produtos químicos no tanque do pulverizador que muitos agricultores têm utilizado para diminuir os custos no controle de pragas.

Além dos aspectos já discutidos para cada técnica é preciso considerar as propriedades físicas e químicas dos produtos, como por exemplo a densidade e a solubilidade que podem influenciar de forma direta os resultados, principalmente na incorporação do produto ao meio e na mistura à calda. Nestes casos, se a calda ou meio ficarem em repouso, pode ocorrer a separação dos produtos e dos esporos por diferentes densidades. Ainda, na mistura com o meio podem ocorrer reações entre os componentes dos meios e dos produtos, alterando a ação dos mesmos sobre o patógeno.

A etiologia dos fungos como as formas de resistência e crescimento saprofítico também devem ser considerados na determinação da técnica mais apropriada para os testes de compatibilidade. Entretanto, a escolha da técnica a ser utilizada in vitro deve ser direcionada para aquelas que reproduzam as condições de campo, onde o produto é aplicado sobre o substrato no qual se encontra o fungo ou onde este virá a se depositar na forma de esporo em aplicações inundativas. Assim, na busca de uma padronização, a pulverização do produto após 
a inoculação do patógeno é a técnica que mais se aproxima das condições de campo.

\section{Parâmetros Utilizados para Avaliar o Efeito} Fungitóxico de Produtos Fitossanitários

Um outro aspecto importante a ser considerado na padronização dos testes de compatibilidade são os parâmetros a serem avaliados. Assim como para as técnicas empregadas, existe uma grande variabilidade nos parâmetros estudados e, consequentemente, na classificação da toxicidade dos produtos.

Nos testes in vitro, alguns autores avaliam somente o crescimento vegetativo e a esporulação do fungo, e em alguns casos também a virulência dos esporos que o fungo desenvolveu em contato com o produto, seguindo a metodologia proposta pela IOBC (HOKKANEN; KOTILUOTO, 1992) e por Alves, Moino e Almeida (1998) ou ainda somente a esporulação (SILVA; SILVA; HEINECK 1993) ou somente o crescimento vegetativo, (CAMARGO, 1983).

Em outros estudos considera-se, além do crescimento vegetativo e produção de esporos, a viabilidade ou a porcentagem de germinação dos esporos em contato com os produtos (AGUDA; ROMBACH; SHEPARD, 1986; BATISTA FILHO et al., 1996; PACHAMUTHU; KAMBLE; YUEN, 1999; NEVES et al., 2001; HIROSE et al., 2001).

Alguns autores discutem e ressaltam a importância de se avaliar a germinação (HIROSE et al., 2001; NEVES et al., 2001; SILVA, 2003), uma vez que é através da germinação que os fungos penetram no corpo dos insetos. Além disso, os conídios germinados tornamse mais expostos e mais vulneráveis à ação dos produtos fitossanitários. Assim, se ocorrer inibição da germinação a eficiência de controle será comprometida, seja em aplicações inundativas ou se o fungo estiver naturalmente presente no agroecossistema e entrar em contato com o produto.

Considerando-se somente o inseto, a germinação e a esporulação são as únicas fases de desenvolvimento em que o fungo está em contato direto com o produto químico quando inseticida, geralmente quando este é pulverizado antes ou após a deposição dos esporos ou sobre os esporos dos insetos mortos pelo fungo, onde ocorreu esporulação. Neste último caso, considerando o inseto como a fonte de nutrientes para o desenvolvimento do patógeno, os produtos somente estarão em contato com os patógenos (esporos) externamente. No caso de fungicidas, insetos que se alimentem de estruturas tratadas com estes produtos, podem absorvê-los e estes passam a circular na sua hemolinfa. Nestes casos, o crescimento vegetativo poderá ser afetado. Assim, para estes produtos deverá ser avaliada a influência dos mesmos no crescimento vegetativo para aqueles que pouco inibirem a germinação. Também neste caso, a esporulação, virulência e viabilidade dos esporos produzidos devem ser avaliadas.

Um outro parâmetro importante, por estar relacionado com a germinação/viabilidade, são as unidades formadoras de colônias (UFC). Este parâmetro, além de indicar um possível atraso ou inibição da germinação (quando a porcentagem de redução das UFC for menor que a da germinação), também pode mostrar uma possível inibição do crescimento vegetativo (quando a porcentagem de redução das UFC for maior que a da germinação) (SILVA, 2003).

\section{Considerações Finais}

A importância da implantação de um protocolo para testes de compatibilidade, entre fungos entomopatogênicos e produtos fitossanitários, é evidente por permitir a comparação de resultados, por disponibilizar informações em programas de manejo fitossanitário onde se pretende preservar um agente de controle natural eliminando a necessidade de testes iniciais de compatibilidade. Assim, dentro de cada programa, os estudos podem concentrar-se e direcionar-se para características específicas do sistema de produção em questão, como tipo de cultivo (perene ou anual), dosagens, intervalos de aplicação 
do fungo e dos produtos, bioecologia do inseto praga, etiologia do patógeno, outros tratamentos fitossanitários de doenças e pragas e as possíveis alternativas para o manejo fitossanitário viabilizando e/ou otimizando a utilização do entomopatógeno dentro da viabilidade econômica da cultura.

Também, estudos utilizando o inseto em questão (in vivo) como indicador da compatibilidade quando isto for possível, (produtos sem ação inseticida) devem complementar e certificar a compatibilidade.

\section{Referências}

AGUDA, R. M.; ROMBACH, M. C.; SHEPARD, B. M. Effect of "neem" oil on germination and sporulation of the entomogenous fungus Metarhizium anisopliae. International Rice Research Newsletter, Manila, v. 11, p. 34-35, 1986.

ALVES, S. B.; RIGITANO R. L. O.; CAMARGO, J. L. G. Avaliação da influência de alguns herbicidas utilizados em cana-de-açúcar e pastagens sobre o fungo Metarhizium anisopliae (Metschnikoff) Sorokin, 1883. Ecossistema, Espirito Santo do Pinhal, v. 5, p. 21-24, 1980.

ALVES, S. B.; MOINO, A.; ALMEIDA, J. E. M. Produtos fitossanitários e entomopatógenos. In: ALVES, S. B.(ed.). Controle Microbiano de Insetos. 2. ed. Piracicaba: FEALQ, 1998. p. 217-238.

ANDERSON, T. E.; ROBERTS, D. W. Compatibility of Beauveria bassiana Isolates with Insecticide Formulations Used in Colorado Potato Beetle (Coleoptera: Chrysomelidae) control. Journal of Economic Entomology, Washington, v. 76, n. 6, p. 1437-1441, 1983.

BATISTA FILHO, A.; LEITE, L. G.; ALVES, E. B.; AGUIAR, J. C. Controle de Cosmopolites sordidus (Coleoptera: Curculionidae) por fipronil e seu efeito sobre Beauveria bassiana. Arquivos do Instituto Biológico, São Paulo, v. 63, n. 2, p. 47-51, 1996.

BATISTA FILHO, A.; ALMEIDA, J. E. M.; LAMAS, C. Effect of thiamethoxam on entomopathogenic microrganisms. Neotropical Entomology, Londrina, v. 30, p. 437-447, 2001.

CADATAL, T. D.; GABRIEL, B. P. Effect of chemical pesticides on the development of fungi pathogenic to some rice insects. The Philippine Entomologist, Laguna, v. 1, n. 5, p. 379-395, 1970.

CAMARGO, L. M. P. C. A. Efeito de alguns piretróides sobre o fungo entomopatogênico Metarhizium anisopliae
(Metsch.) Sorokin. Biológico, São Paulo, v. 49, n. 3, p. $65-$ $68,1983$.

CORREIA, A. C. B; BERNARDO, E. R. A.; SILVA, R. A.; PAZINI, W. C. Compatibilidade de agrotóxicos com o fungo entomopatogênico Paecilomyces fumosoroseus (Wise) Brown \& Smith in vitro. In: SIMPÓSIO DE CONTROLE BIOLÓGICO, 7, 2001, Poços de Caldas. Resumos... Poços de Caldas: P. J. Eventos, 2001. p. 113.

DOUGHERTY, E. M.; REICHELDERFER, C. F.; FAUST, R. M. Sensitivity of Bacillus thuringiensis var. thuringiensis to various insecticides and herbicides. Journal of Invertebrate Pathology, New York, v. 17, p. 292-293, 1971.

HALL, I. M.; DUNN, P. H. The effect of certain insecticides and fungicides on fungi pathogenic to the spotted alfafa aphid. Journal of Economic Entomology, Washington, v. 52, p. 28-29, 1959.

HIROSE, E.; NEVES, P. M. O. J.; ZEQUI, J. A.; MARTINS, C. L. H.; PERALTA, C. H.; MOINO, A. Effect of biofertilizers and Neem Oil on the Entomopatogenic fungi Beauveria bassiana (Bals.) Vuill. and Metarhizium anisopliae (Metsch.) Sorok. Brazilian Archives of Biology and Technology, Curitiba, v. 44, n. 4, p. 419-423, 2001.

HOKKANEN, H. M. T.; KOTILUOTO, R. Bioassay of sideeffects of pesticides on Beauveria bassiana and Metarhizium anisopliae, standardized sequencial testing procedure. In: WORKING GROUP PESTICIDES AND BENEFICIAL ORGANISMS, 1992.p. 148-151.

IGNOFFO, C.M.; HOSTETTER; GARCIA, D. L.; PINNELL, C. R. R. Sensivity of the entomopathogenic fungus Nomurae rileyi to chemical pesticides used on soybeans. Environmental Entomology, Lanham, v. 4, n. 5, p. $765-$ $768,1975$.

JAQUES, R. P.; PATTERSON, N. A. Control of the apple sucker, Psylla mali Schmidb., by the fungus Entomophthora sphaerosperma (Fresenius). Canadian Entomologist, Ottawa, v. 94, p. 818-825, 1962.

JARUS-SU, J.; GRODEN, E.; ZHANG, J. Effects of selected fungicides application on Beauveria bassiana-induced mortality of the Colorado potato beetle (Coleoptera: Chrysomelidae). Biological Control, San Diego, v. 15, n. 3, p. 259-269, 1999.

LORIA, R.; GALAINI, S.; ROBERTS, D. W. Survival of inoculum of the entomopathogenic fungus Beauveria bassiana as influenced by fungicides. Environmental Entomology, Lanham, v. 12, n. 6, p. 1724-1726, 1983.

MALO, A. R. Estudio sobre la compatibilidad del hongo Beauveria bassiana (Bals.) Vuill. con formulaciones comerciales de fungicidas e insecticidas. Revista Colombiana de Entomologia, Bogotá, v. 19, p. 151-158, 1993. 
MOINO, A.; ALVES, S. B. Efeito de imidacloprid e fipronil sobre Beauveria bassiana (Bals.) Vuill. e Metharhizium anisopliae (Metsch.) Sorok. no comportamento de limpeza de Heterotermes tenuis (Hagen). Anais Sociedade Entomológica do Brasil, Londrina, v. 27, n. 4, p. 611-619, 1998.

NEVES, P. M. O. J.; HIROSE, E.; TCHUJO, P. T.; MOINO, A. Compatibility of entomopathogenic fungi with neonicotinoids insecticides. Neotropical Entomology, Londrina, v. 30, n. 2, p. 263-268, 2001.

OLIVEIRA, C. N.; NEVES, P. M.O. J.; KAWAZOE, L. S. Compatibility between the entomopathogenic fungus Beauveria bassiana and insecticides used in coffee plantations. Scentia Agrícola, Piracicaba, v. 60, n. 4, p. 663-667, 2003.

OLMERT, I.; KENNETH, R. G. Sensitivity of the entomopathogenic fungi Beauveria bassiana, Verticillium lecanii and Verticillium sp. to fungicides and insecticides. Environmental Entomology, Lanham, v. 3, p. 33-38, 1974.

PACHAMUTHU, P.; KAMBLE, S. T.; YUEN, G. Y. Virulence of Metharhizium anisopliae (Deuteromycotina: Hyphomycetes) strain ESC-1 to the German Cockroach (Dictyoptera: Blattelidae) and its compatibility with insecticides. Journal of Economic Entomology, Washington, v. 92, n. 2, p. 340-346, 1999.
POPRAWSKI, T. J.; MAJCHROWICZ, I. Effects of herbicides on in vitro growth and sporulation of entomopathogenic fungi. Crop Protection, Oxford, v. 14, p. 81-87, 1995.

SILVA, L.; SILVA, R. F. P.; HEINECK, M. A. Avaliação “in vitro" do efeito de diferentes inseticidas sobre a esporulação do fungo Nomuraea rileyi (Farlow) Samson. Anais da Sociedade Entomológica do Brasil, Itabuna, v. 22,p. 99-103, 1993.

SILVA. R. Z. Comparação de técnicas e parâmetros utilizados em testes de compatibilidade entre Beauveria bassiana (Bals.) Vuill. e produtos fitossanitários in vitro. 2003. 57 f. Dissertação (Mestrado em Agronomia) Programa de Pós-graduação. Universidade Estadual de Londrina, Londrina, 2003.

TODOROVA, S. I.; CODERRE, D.; DUCHESNE, R. M.; CÔTÉ, J. C. Compatibility of Beauveria bassiana with selected fungicides and herbicides. Environmental Entomology, Lanham, v. 27, n. 2, p. 427-433, 1998. 